\title{
A Brief Tutorial on Manuscript Preparation: From Journal Selection to Manuscript Review
}

Christopher N. Ochner, PhD, 1,2 Jocelyn Mineo ${ }^{1,2}$

\section{Abstract}

\section{Description}

This article is designed to introduce the novice researcher to the process of journal selection, manuscript submission and manuscript review. PubMed indexing, journal readership, scope, focus, impact factor, fees and acceptable manuscript types are discussed in the first section. The remainder of this article focuses on manuscript preparation, submission and review, including formatting, pre-submission inquiry, submission portals, and the manuscript review process. Specific recommendations are provided to assist the reader in navigating these stages.

Author affiliations are listed at the end of this article.

Correspondence to: Dr. Christopher Ochner 450 East Las Olas Blvd, Suite 1100 Fort Lauderdale, FL 33301 (Christopher.Ochner@ hcahealthcare.com)

\section{Keywords}

publication; GME; manuscript; journal selection; scholarly communication; journal impact factor

\section{Introduction}

Several factors need to be taken into consideration when attempting to bring a manuscript from preparation to publication in a reputable academic journal. For authors unfamiliar with this process, it may seem daunting. This article is designed to familiarize the reader with the journal selection, manuscript submission and manuscript review processes. Although written primarily for inexperienced authors, some suggestions offered in this article may help even seasoned authors maximize the probability of manuscript acceptance. With regard to journal selection, PubMed indexing, journal readership, scope, focus, impact factor, fees and acceptable manuscript types are discussed. The remainder of this article focuses on manuscript preparation, submission and review. In this latter section, formatting, presubmission inquiry, submission portals and the manuscript review process are discussed in detail. Specific recommendations are provided to assist the reader in navigating these stages and considering factors relevant to each. This article should serve as a guide for new authors and those with relatively little authorship experience, as well as a collection of techniques of which more experienced authors may also be able to take advantage.

\section{Journal Selection}

Journal selection is discussed prior to manuscript preparation in this article, as these authors recommend identifying several appropriate journals to which the manuscript may be submitted prior to completing the manuscript. This is for two main reasons, both stemming from the strong recommendation to conduct what is called a "presubmission inquiry" (discussed in the second half of this manuscript) prior to submitting an article for publication. First, it will be easier and faster to format the manuscript for the journal to which it is to be submitted prior to completion. Second, the Editor-in-Chief of a target journal may be interested in an article but ask the author to pivot and make changes to the article, which is easier to do prior to completion. A number of relevant factors should be taken into consideration when gathering a short list of journals to which to potentially submit a manuscript, discussed below.

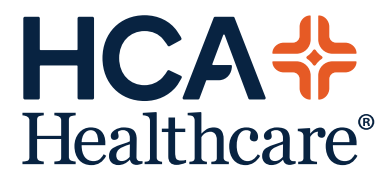

www.hcahealthcarejournal.com

(C) 2020 HCA Physician Services, Inc. d/b/a Emerald Medical Education
HCA Healthcare Journal of Medicine 


\section{PubMed indexing}

The first, and potentially most important, factor discussed is that of PubMed indexing. In 1996, the National Library of Medicine established the PubMed Index system.' With the exception of new journals that have not yet had the opportunity to earn PubMed indexing, authors should look to publish in journals that are indexed on PubMed, as this is a quick litmus test of journal legitimacy. Journals indexed on PubMed can generally be considered to be reputable, peer-reviewed academic journals and journals not indexed on PubMed can generally be considered to be less reputable and/or nonpeer reviewed, again, with the exception of new journals that are yet to be indexed. ${ }^{2}$ Although far from a perfect indicator due to a number of exceptions, the vast majority of reputable, peer-reviewed journals are indexed on PubMed and very few (if any) illegitimate or non-peer reviewed journals are indexed on PubMed. PubMed indexing is, in fact, so important that the Accreditation Council on Graduate Medical Education considers PubMed-indexed publications to be one of the highest forms of scholarly activity achievable, it considers nonPubMed-index publications to be on par with poster presentations. PubMed indexing can be checked by going to PubMed and searching the name of the journal. If articles from that journal appear on PubMed, the journal is likely PubMed indexed.

\section{General readership vs. targeted audience}

General readership journals cover an extensive array of topics and appeal to a broad audience. For example, journals such as Nature and Science could potentially contain articles covering any aspect of nature or science. Similarly, their readership could be anyone interested in nature or science, a very general readership. Conversely, targeted journals typically publish articles relevant only to a particular specialty or subspecialty. The Journal of Cardiovascular Magnetic Resonance, for example, is clearly a more esoteric journal, which publishes articles relating only to this specialty and is likely read only by individuals with some interest in this particular specialty. There are exceptions, but general readership journals tend to be more selective in accepting manuscripts for publication. Thus, unless an article has very clear broad-based ap- peal, authors are likely better off submitting to a targeted journal focused on the area related to the content of the article.

\section{Journal focus}

Journals often prefer to publish manuscripts that are either more clinically oriented or more research oriented. Most journals will not state this overtly, but authors can determine the journal focus (clinical practice vs. research) by looking at the types of articles accepted and the titles of articles recently published. If only review and original research articles are accepted and recently published articles are more academic in nature, with heavy emphasis on precise methodology and statistics, the journal likely has a research focus. The Editor-in-Chief of these journals will likely have a PhD as opposed to an MD or DO degree. If the journal accepts case reports and most of the articles appear to be relevant to clinical practice, the journal likely has more of a clinical orientation. The Editor-in-Chief of these journals will likely have an MD or DO, as opposed to a PhD degree. Overall, journals with a clinical orientation are more accepting of, and more likely to publish, articles relevant to clinical practice.

\section{Journal scope}

Every journal website lists the scope of that journal, which informs potential authors about the range of topics that journal thinks would be of interest to its readership and is willing to publish. In order to increase the chances of publication, a manuscript should only be submitted to a journal if it fits within the scope of the journal. If a manuscript falls outside the stated scope of a particular journal, it is better submitted to a more appropriate journal, as journals very rarely make exceptions in accepting manuscripts with topics that fall outside their stated scope.

\section{Types of manuscripts accepted}

Journals only accept particular types of articles, which will be listed on the journal's website, typically in the author guidelines. Some journals will accept brief reports, or short reports, which are similar to original research manuscripts, but brief reports have strict length limits. This type of manuscript is suitable for preliminary studies and small-scale research. Less than $50 \%$ of journals accept 
case reports, and even fewer journals accept mini-reviews. If an author is writing a case report or mini-review manuscript, they may have to spend some time finding the relatively few journals that are relevant to the topic they are writing about and accept the type of manuscript they are composing.

\section{Impact factor}

The impact factor of a journal describes its overall influence. Technically, it is calculated by an algorithm that assesses how many times articles published in that journal are cited by articles published in other PubMed indexed journals. ${ }^{3}$ As the name implies, the impact factor serves as a marker of how large of an impact the articles published in a particular journal have on its content area but can also be thought of as an indicator of the breadth of a journal's reach. Impact factor ranges from 0.1 for journals that are less reputable up to around 80 for the New England Journal of Medicine. There are a considerable number of journals with impact factors below 3.0 and relatively few journals with an impact factor 10.0 or higher. ${ }^{4}$ The higher the impact factor, the more selective the journal, and the lower the likelihood of a submitted manuscript being published. The perceived impact of the manuscript should be commensurate with the impact factor of the journal to which an author submits their manuscript. In the experience of these authors, the majority of case reports and articles describing smaller research or quality improvement projects are published in journals with impact factors below 3.0 (usually 0.5 to 2.0). In the opinion of these authors, it is better to have several publications in lower impact journals than it is to have one publication in a higher-impact journal, so manuscripts should be submitted to the highest quality journal that the authors believe is very likely to publish the manuscript. If a journal does not have an impact factor, authors should check to confirm the journal is indexed on PubMed or if the journal is new before deciding to submit to that particular journal. New journals will not have an impact factor because it takes up to three years to calculate.

\section{Submission and/or publication fees} Journals charging a submission fee for a man- uscript is rare. If encountered, authors should consider removing that journal from their list of candidate journals. An increasing number of legitimate journals are charging a publication fee, but there are also less reputable "open access" journals that charge $\$ 1500$ to $\$ 3000$ or more to publish articles. These journals, known as "predatory journals," 5 are rarely indexed on PubMed and should generally be avoided. It should be noted that not all open access journals are predatory journals, as there are a number of reputable and PubMed-indexed, open access journals (e.g., PLOS Medicine); however, nearly all predatory journals are open access journals.

\section{Manuscript Preparation, Submis- sion and Review}

The manuscript preparation, submission and review process can be intimidating for inexperienced authors. This section reviews and provides essential tips to navigate this process in a way that maximizes the chance of manuscript publication. Primarily because it is a challenging and time-consuming process, publication in PubMed-indexed journals is considered one of the highest forms of scholarly activity a resident, fellow or graduate medical education faculty member can achieve, and it is particularly important for residents seeking fellowship.

\section{Manuscript preparation}

The first step in preparing a manuscript for submission is to read the author instructions for the journal to which the author plans to submit and follow the instructions exactly. Most journal websites will have a page that includes the journal's preferred style for citations and references, and limits on the number of pages, words, references, tables and figures. The manuscript should be set up in the appropriate order, which is as follows: (1) title page, (2) abstract, (3) body, (4) references, (5) tables, (6) figures and (7) appendices. Tables and figures should be located after the references, not embedded in the text.

To begin manuscript preparation, experienced authors often create an outline, which summarizes the information to be included in each section of the manuscript. The outline serves as a tool in assisting authors to adhere to the 
conventions of academic manuscripts, which are to include all relevant detail but no excess information or colloquialisms. ${ }^{6}$ This uniformity allows readers to derive sought-after information from the manuscript in minimal time.

\section{Presubmission inquiry}

Authors can only submit a manuscript to one journal at a time, however, it is possible to gauge interest in a manuscript from multiple journals at once by submitting several presubmission inquiries. A presubmission inquiry allows authors to contact editors to inquire whether they may be interested in their article, since it is the editors that ultimately decide which articles to publish. Editors do not always respond but, when they do, it is a useful tool to get feedback from multiple journals simultaneously regardless of whether the response is positive or negative.

Using their short list of appropriate journals, authors should search the website of each journal to find information about the editorial team and locate the name of the Editor-in-Chief. Their email address might be listed, but authors may have to search the web to find a recent publication of theirs on PubMed. If the Editor-in-Chief is listed as the corresponding author, their email address will be listed. Authors can then send a brief message with the abstract of their manuscript, which reads as follows:

\section{Dear Dr. [NAME OF EDITOR-IN-CHIEF],}

I apologize for the unsolicited email, but my colleagues and I are working on a manuscript about [TOPIC] that we thought might be of interest to your readership. The abstract is below. Would you please let me know if you would be interested in having this article reviewed for possible publication in [JOURNAL NAME]? Your feedback is greatly appreciated. Sincerely,

A positive response would be if the editor indicates that the manuscript may be appropriate for their readership and encourages submission. Negative responses are also useful because authors do not lose time submitting to one journal and waiting for a response before submitting to another journal.

\section{Manuscript Submission}

Most journal websites will have a link to the manuscript submission portal. Authors typically have to register for an account if they have never submitted a manuscript to that journal before. Once registered, the system should provide step-by-step instructions for the submission process, which should be followed exactly.

Authors are often asked to upload the cover letter, manuscript body, tables and figures as separate files. The cover letter is sometimes optional, but it is recommend to submit a brief cover letter with the following regardless of whether it is required:

Dear Dr. [NAME OF EDITOR], We are pleased to submit this [TYPE OF MANUSCRIPT] for publication in [JOURNAL NAME]. This manuscript represents original work, and all authors have approved the final version for submission. None of the authors have anything to disclose [IF THIS IS TRUE, OTHERWISE DESCRIBE ANY POTENTIAL CONFLICTS OF INTEREST]. The presented manuscript has not been previously published and is not under consideration anywhere else. Please let me know if there are any questions concerning this manuscript.

Sincerely,

Some experienced authors also suggest voluntarily including the names and emails for suggested reviewers and/or any reviewers that the authors feel should be avoided (e.g., someone known to have a rivaling opinion). In some rare instances, journals mandate reviewer suggestions. In this circumstance, that information will typically be entered into the submission system itself but may be included in the cover letter. If authors are unsure of how to identify appropriate experts in the area for their article, they should look at the names of the authors cited in the Introduction of the article.

The manuscript body usually contains the title page, abstract, body and references together in that order. Some journals want tables included at the end of the manuscript file, while others prefer them to be uploaded separately. Similarly, some journals want figure captions included with the manuscript file, while others want them included in a separate file. Discrepancies 
among formatting preferences is the reason it is imperative to review the author instructions carefully.

\section{Manuscript Review Process}

Following submission, manuscripts are assigned an editor. All journals have a certain number of editors and usually only one editor-in-chief. Each article will be assigned to an editor, who manages the review and decision process. This editor will typically ask an associate, or junior editor, to do an internal review of the manuscript to confirm the following: (1) the manuscript is a type of article the journal accepts; (2) the topic falls within the scope of the journal and would be of interest to the journal readership; (3) the overall quality of the manuscript and potential impact of the results are high.

If the article does not meet one of these criteria, it is likely to be rejected right away, which is referred to as an "editorial reject." If the article receives an editorial reject, authors will typically be notified within about two weeks of submission. ${ }^{7}$ If authors do not receive a response from the journal for more than three weeks, their manuscript has likely passed the editorial review stage. If the article meets the above cri- teria and has passed the editorial review stage, it will be sent out for external review.

External review involves the solicitation of recognized experts on the subject matter of the submitted article, which are usually researchers who have many publications on the topic of the submitted manuscript. These may be experts who published articles cited in the introduction of the submitted manuscript. These experts will be invited to review the article, offer feedback and make a recommendation to the editor about whether the journal should publish the article (Figure 1). Most manuscripts sent for external review will be reviewed by two or three reviewers, but that number can range from one to five. The editorial team will take about two to three weeks to identify the appropriate experts, invite them to review the manuscript and receive a response, though this timeline can vary dramatically. These external reviewers will take an additional three to four weeks to offer a recommendation on the manuscript. Again, however, the time required for this can vary substantially. The external reviewers will provide specific criticisms of the manuscript and provide one of the following recommendations to the editor about the disposition of the article:

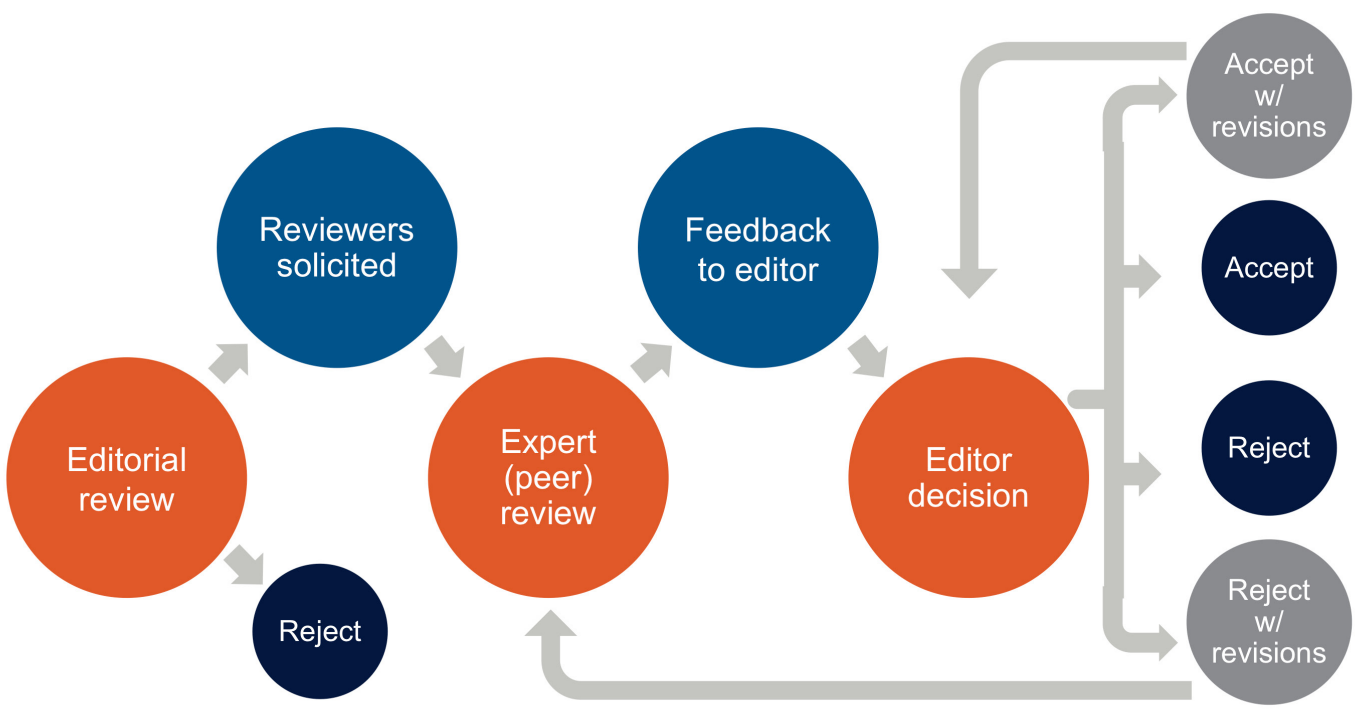

Figure 1. The Manuscript Review Process

External reviewers will write out specific criticisms of the manuscript and provide recommendations to the editor about the disposition of the article. 
- Reject without opportunity to revise: If au thors receive this, they should submit their manuscript to the next journal on their short list.

- Reject with minor revisions: This is also known as "revise and resubmit," which indicates that there were minor criticisms that need to be addressed. The reviewers do not believe the manuscript should be accepted in its current form, but the authors should be given the opportunity to address the minor criticisms.

- Reject with major revisions: This is similar to "reject with minor revisions," but the criticisms are major and must be completely addressed for the reviewers to recommend acceptance.

- Accept with minor revisions: This is less common, but it is still used occasionally. This recommendation indicates that the authors need to make only a few very minor changes for the reviewers to deem it ready for publication.

- Accept with no revisions: This means that the journal is accepting the manuscript exactly as submitted, which is rare.

Ultimately, the editor makes the final decision about the disposition of the manuscript, but they will follow the recommendations of the external reviewers the vast majority of the time. They may use their own discretion when reviewers make different recommendations about the article. This is not uncommon, and a third reviewer will often be asked to offer an opinion if there were only two external reviewers initially.

If authors receive a reject with the opportunity to revise and resubmit their manuscript, they will generally want to take that opportunity. Only in very rare instances where a reviewer wants a major revision, such as redoing all of the analyses or rewriting the entire manuscript, would it be worth starting the submission process over with a different journal. Although they are similar, receiving an 'accept with minor revisions' is different from receiving a 'reject with minor revisions.' If an author receives an 'accept with minor revisions,' the publication can be added to their curriculum vitae (or residency/fellowship application) with the disposition of "accepted," which can make a difference for medical students applying for residency and residents applying for fellowship. The best way to ensure the manuscript gets published is to thoroughly address all reviewer comments and submit a revised manuscript with a separate list of each reviewer criticism and the authors' response to each of them. It is not uncommon to disagree with particular comments and/or reviewer perspectives. In these instances, it is vital to address the reviewer in a professional manner and either adhere to the request or detail why you disagree and substantiate your position with evidence (i.e., references). Note, however, that most experts suggest adhering to most reviewer suggestions unless the authors feel the change would substantively reduce the accuracy or impact of the manuscript.

The revised manuscript and list of responses to criticisms will be sent back out to the reviewers, almost always the same reviewers that provided the initial feedback. They will evaluate whether the authors adequately addressed all criticisms and make a recommendation to the editor about whether the article should be accepted for publication. If the authors have done well in addressing reviewer criticisms, most of the time the article will be accepted. However, it is possible that further revisions may be requested or that the manuscript is rejected without the opportunity to revise again. If more revisions are required, the same process and disposition options repeat until the manuscript reaches one of the two terminal dispositions (i.e., reject without opportunity to revise or accept without revisions).

Note that most reviews are single-blind, meaning that the identity of the reviewers will not be revealed to the authors, but the authors' identities will be revealed to the reviewers. However, some journals practice double-blind review, where neither the authors nor reviewers will know the identity of the other party. For double-blind reviews, authors will likely be asked to submit a version of the manuscript that does not contain their names. There are even a few journals that do not blind the names of the authors or reviewers.

For articles sent for external review, this entire process can take from one to nine months, with an average of three to four months, including time for review and revision. ' For this reason, some authors become frustrated with this 
process and many high-quality manuscripts become "orphaned," even when they may have been quite close to acceptance for publication. For all, but especially new authors, we stress patience and perseverance through this process. The rewards of publication can be great and many.

\section{Conclusion}

Although it typically requires years of experience, and much trial and error, to truly become an expert in the process of manuscript publication, the techniques described in this article should demystify the process for novice authors and provide some additional insight for more seasoned authors. In preparing a manuscript for publication, remember to include all important and relevant detail, but no unnecessary wording. Avoid colloquialisms and imprecise language. The writing should be specific and purposeful, adhering to all relevant conventions for scientific publication. Make sure that anything not considered axiom, or general knowledge, is properly cited and referenced. Software, such as EndNote, Microsoft Word, Zotero and Paperpile may be helpful in formatting references. After completing a compelling abstract, create a short list of appropriate journals and solicit editor feedback on the abstract from each one. Upon choosing the first (and hopefully only) journal to which to submit a manuscript, follow the instructions for authors on the journal's website precisely. Many reviewers consider imprecision in formatting, grammar and spelling in a manuscript as a reflection of imprecision in the way the project was conducted and the manuscript written. Remember, the peers (experts) who review articles only make suggestions about whether the article should be published to the editor assigned to that article, who makes the ultimate decision. Except in very rare circumstances where the authors feel they would be unable to adequately address reviewer criticisms, an article that is rejected with the opportunity to revise (i.e., reject with minor or major revisions) should be carefully revised according to reviewer instruction and resubmitted within a month (or sooner if specified by the journal). Revised manuscripts that are not resubmitted in a timely manner may be treated as a new submission and sent to different reviewers who may look at it less favorably than the first set of reviewers. As long as revisions address all reviewer criticisms, the vast majority of "revise and resubmit" articles wind up being accepted and published. Overall, the process is generally lengthy and can be trying at times, but publication in a PubMed-indexed journal is considered one of the highest forms of scholarly activity, and can be highly rewarding for those willing to put in the time and effort.

\section{Conflicts of Interest}

The authors declare they have no conflicts of interest.

Dr. Christopher Ochner and Jocelyn Mineo are employees of HCA Physician Services Group, a company affiliated with the journal's publisher.

This research was supported (in whole or in part) by HCA Healthcare and/or an

HCA Healthcare affiliated entity. The views expressed in this publication represent those of the author(s) and do not necessarily represent the official views of HCA Healthcare or any of its affiliated entities.

\section{Author Affiliations}

1. HCA Healthcare: East Florida Division GME, Ft. Lauderdale, FL

2. HCA Physician Services Group, East Florida Division, Ft. Lauderdale, FL

\section{References}

1. Canese K, Weis S. PubMed: The Bibliographic Database. The NCBI Handbook [Internet]. 2nd edition. https://www.ncbi.nlm.nih.gov/books/ NBK153385/. Published March 20, 2013. Accessed December 12, 2019.

2. Manca A, Moher D, Cugusi L, Dvir Z, Deriu F. How predatory journals leak into PubMed. CMAJ. 2018;190(35):E1042-E1045. https://doi. org/10.1503/cmaj.180154

3. Alberts B. Impact factor distortions. Science. 2013;340(6134):787. https://doi.org/10.1126/science. 1240319

4. Leydesdorff L. Alternatives to the journal impact factor: 13 and the top-10\% (or top-25\%?) of the most-highly cited papers. Scientometrics. 2012; 92(2):355-365. https://doi.org/10.1007/ s11192-012-0660-6

5. Beall J. Predatory publishers are corrupting open access. Nature. 2012;489(7415):179. https://doi. org/10.1038/489179a

6. Bhargava P. The KISS Principle in Academic Writing. Curr Probl Diagn Radiol. 2015;44(6):473. 
https://doi.org/10.1067/j.cpradiol.2015.08.005

7. Huisman J, Smits J. Duration and quality of the peer review process: the author's perspective. Scientometrics. 2017;113(1):633-650. https://doi. org/10.1007/s11192-017-2310-5 